\title{
Achilles Tendon Length Is Not Related to 100-m Sprint Time in Sprinters
}

\author{
Daichi Tomita, Tadashi Suga, Hiromasa Ueno, Yuto Miyake, Takahiro Tanaka, Masafumi Terada, \\ Mitsuo Otsuka, Akinori Nagano, and Tadao Isaka \\ Ritsumeikan University
}

\begin{abstract}
This study examined the relationship between Achilles tendon (AT) length and 100-m sprint time in sprinters. The AT lengths at 3 different portions of the triceps surae muscle in 48 well-trained sprinters were measured using magnetic resonance imaging. The 3 AT lengths were calculated as the distance from the calcaneal tuberosity to the muscle-tendon junction of the soleus, gastrocnemius medialis, and gastrocnemius lateralis, respectively. The absolute 3 AT lengths did not correlate significantly with personal best $100-\mathrm{m}$ sprint time $(r=-.023$ to .064 , all $P \mathrm{~s}>.05)$. Furthermore, to minimize the differences in the leg length among participants, the 3 AT lengths were normalized to the shank length, and the relative 3 AT lengths did not correlate significantly with personal best $100-\mathrm{m}$ sprint time $(r=.023$ to .102 , all $P \mathrm{~s}>.05)$. Additionally, no significant correlations were observed between the absolute and relative (normalized to body mass) cross-sectional areas of the AT and personal best 100-m sprint time $(r=.012$ and .084 , respectively, both $P \mathrm{~s}>.05$ ). These findings suggest that the AT morphological variables, including the length, may not be related to superior $100-\mathrm{m}$ sprint time in sprinters.
\end{abstract}

Keywords: ankle joint, tendon cross-sectional area, triceps surae muscle, stretch-shortening cycle, stiffness, magnetic resonance imaging

Superior sprint performance is achieved using gross torques of the lower limb joints ${ }^{1}$ potentially by increasing peak vertical ground reaction force. ${ }^{2,3}$ The Achilles tendon (AT) plays an important role in storing and returning elastic energy during the stance phase of human locomotion, including sprinting. ${ }^{4}$ Thus, the favorable AT morphology may increase in the ankle plantar flexor torque and peak vertical ground reaction force and may help achieve superior sprint performance. ${ }^{4,5}$ Using magnetic resonance imagining (MRI), we and others previously reported a positive correlation between longer AT length and better running performance in endurance runners. ${ }^{6,7}$ Furthermore, one study by Monte and Zamparo ${ }^{8}$ determined that longer AT correlated with better sprint performances, assessed using personal best 100-m sprint time and sprint variables during a $20-\mathrm{m}$ sprint in sprinters. The longer AT may be an important morphological factor in achieving superior performance in sprinters, similar to endurance runners, which may be due to an increase in the plantar flexor torque and peak vertical ground reaction force. Although the study of Monte and Zamparo ${ }^{8}$ measured the AT length using ultrasonography (US), MRI is more appropriate for measuring morphological variables, ${ }^{9,10}$ including the AT length. ${ }^{6,7}$ Additionally, although they measured only the AT length of the gastrocnemius medialis $\left(\mathrm{GM}_{\mathrm{AT}}\right)$, the AT length can be measured from 3 different portions of the triceps surae, including the soleus $\left(\mathrm{SOL}_{\mathrm{AT}}\right)$ and gastrocnemius lateralis $\left(\mathrm{GL}_{\mathrm{AT}}\right){ }^{7}$ Therefore, the impact of the AT length on sprint performance has not been fully understood.

The plantar flexor torque is a major source of torques required for body support and propulsion during the stance phase while sprinting. ${ }^{1}$ However, previous studies determined that although the

The authors are with the Faculty of Sport and Health Science, Ritsumeikan University, Kusatsu, Shiga, Japan. Suga (t-suga@fc.ritsumei.ac.jp) is corresponding author. plantar flexor torque was increased by changing velocity from walking to running, it remained unchanged when altering velocity from running to sprinting. ${ }^{11,12}$ Thus, the plantar flexor torque may not play an important role in increasing peak vertical ground reaction force and achieving superior sprint performance during 100-m sprinting; in particular, a longer AT is not required for better sprint performance. Based on this background, we hypothesized that the AT length might not be related to sprint performance in sprinters. To test this hypothesis, using MRI, we examined the relationships between 3 AT lengths of the triceps surae and 100-m sprint time in sprinters.

\section{Methods}

\section{Participants}

Forty-eight well-trained male sprinters (age, 20.7 [1.9] y) participated in this study. To determine the required sample size of this study, we used 2 effect sizes $(0.614$ and 0.495 , respectively, for the knee extensor moment arm and forefoot bone length) of the relationships between the morphological variables and personal best 100-m sprint time in sprinters on our previous studies. ${ }^{13,14}$ The $\alpha$ and $\beta$ levels employed 0.05 and 0.2 (80\% power), respectively. The calculated necessary number of subjects was 18 to 29 (ie, 29); thus, we considered that the number of participants recruited in this study was sufficient for ensuring statistical power and sensitivity. The participants were involved in regular sprint training at least 5 times per week and had regular competition. Their personal best time of a $100-\mathrm{m}$ race within the past 1 year before the measurement of this study ranged from 10.22 to 11.86 seconds (mean, 11.12 [0.43] s). All participants were informed of the experimental procedures and provided written consent to participate in this study. This study was approved by the ethics committee of Ritsumeikan University (BKCIRB-2016-047). 
MRI

The MRI measurement was performed using a 1.5-T magnetic resonance system (Signa HDxt; GE Medical Systems, Milwaukee, WI). To measure cross-sectional area (CSA) and 3 lengths of the AT, participants were placed in a supine position on the scanner bed, with both legs fully extended and both ankles set at the neutral position (ie, $0^{\circ}$ ).

Representative sagittal and axial images for calculating 3 lengths and CSA of the AT on MRI are shown in Figure 1. With regard to the AT length measurement, $3 \mathrm{D}$ isotopic $T_{1}$-weighted MRI scans of the lower leg were acquired with 8-channel coil. Sagittal scans were obtained in success slices with a repetition time of 10.4 milliseconds, echo time of 3.3 milliseconds, slice thickness of $1 \mathrm{~mm}$, field of view of $38 \mathrm{~cm}$, and matrix size of $288 \times 288$ pixels. The AT lengths at 3 individuals muscles of the triceps surae were calculated as the distances from the calcaneal tuberosity to the muscle-tendon junction of the SOL (ie, $\mathrm{SOL}_{\mathrm{AT}}$ ), GM (ie, $\mathrm{GM}_{\mathrm{AT}}$ ), and GL (ie, $\mathrm{GL}_{\mathrm{AT}}$ ), respectively, as in our previous study. ${ }^{7}$ To minimize differences in the leg length among participants, the AT lengths for each muscles were normalized to the shank length., ${ }^{7,15}$ The shank length was measured using a tape measure and defined as the distance from the proximal head of the fibula to the tip of the lateral malleolus. ${ }^{7,15}$ In addition to the absolute AT lengths, the relative AT lengths were used for the analysis of this study.

With regard to the AT CSA measurement, axial $T_{1}$-weighted MRI scans of the lower leg were acquired with 8-channel coil. Axial scans were obtained in successive slices with an interdistance of $5 \mathrm{~mm}$ from the muscle-tendon junction of the SOL to the calcaneal tuberosity with a repetition time of 600 milliseconds, echo time of 7.7 milliseconds, slice thickness of $5 \mathrm{~mm}$, field of view of $380 \mathrm{~mm}$, and matrix size of $512 \times 256$ pixels. The CSA of the AT was calculated as an average of 10,20 , and $30 \mathrm{~mm}$ above the distal insertion of the AT, as in our previous study. ${ }^{7}$ To minimize differences in body size among participants, the AT CSA was normalized to body weight to the two-third power. ${ }^{7,16,17}$ In addition to the absolute AT CSA, the relative AT CSA was used for the analysis of this study.

\section{Sagittal image}
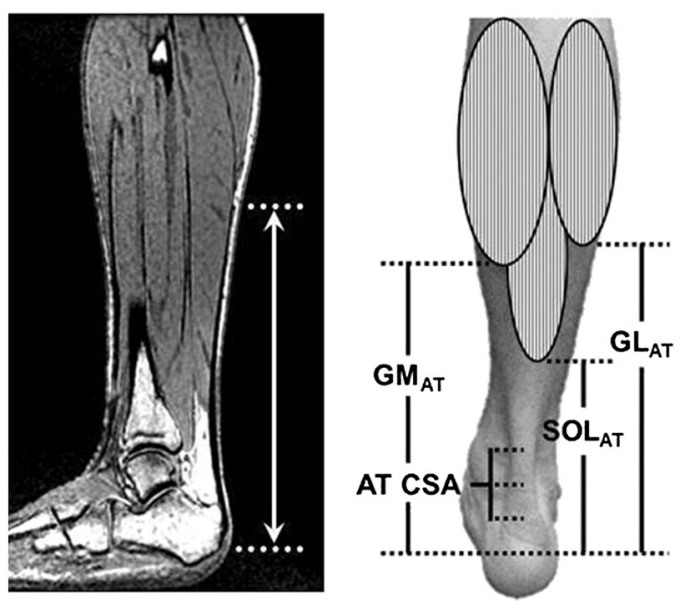

The analyses for measuring the lengths and CSA of the AT were conducted using image analysis software (OsiriX version 5.6; OsiriX Foundation, Geneva, Switzerland). Each measurement was performed twice, and the mean of the 2 measurements was used for the analysis of this study. With regard to the 3 AT lengths, coefficient of variations (CVs) of 2 measurements for the $3 \mathrm{AT}$ lengths in all participants were $0.9 \%(0.7 \%)$ for $\mathrm{SOL}_{\mathrm{AT}}, 0.3 \%$ $(0.3 \%)$ for $\mathrm{GM}_{\mathrm{AT}}$, and $0.4 \%(0.2 \%)$ for $\mathrm{GL}_{\mathrm{AT}}$, respectively. Intraclass correlation coefficients (ICC) of 2 measurements for each AT length were .999 (95\% confidence interval [CI], .995-.999) for $\mathrm{SOL}_{\mathrm{AT}}, .999$ (95\% CI, .998-.999) for $\mathrm{GM}_{\mathrm{AT}}$, and .999 (95\% CI, .997-.999) for $\mathrm{GL}_{\mathrm{AT}}$, respectively. With regard to the AT CSA, CV of 2 measurements for the AT CSA (ie, mean AT CSA) in all participants was $0.5 \%(0.5 \%)$. ICC of 2 measurements for the AT CSA was .999 (95\% CI, .998-.999).

To further assess the reproducibility of the AT morphological variable measurements obtained in the present study, in a preliminary study, we measured 3 lengths and CSA of the AT on 2 separate days in 15 healthy young men (age, 21.9 [1.1] y; body height, 170.6 [4.1] cm; body weight, 64.5 [7.6] kg). ICCs of the 3 AT lengths and CSA on the 2 days were .985 (95\% CI, .957 .995) for $\mathrm{SOL}_{\mathrm{AT}}, .984$ $\left(95 \%\right.$ CI, .953-.994) for $\mathrm{GM}_{\mathrm{AT}}, .991(95 \% \mathrm{CI}, .973 .997)$ for $\mathrm{GL}_{\mathrm{AT}}$, and .984 (95\% CI, .953-.995) for AT CSA, respectively.

\section{Statistical Analysis}

All data are presented as mean (SD). Relationships between the AT morphological variables and personal best $100-\mathrm{m}$ sprint time were assessed using a Pearson's product-moment correlation. Statistical significance level was defined at $P<.05$. All statistical analyses were conducted using SPSS (version 19.0; IBM Corp, Armonk, NY).

\section{Results}

Mean values of physical characteristics and AT morphological variables in sprinters are listed in Table 1 . There were no significant

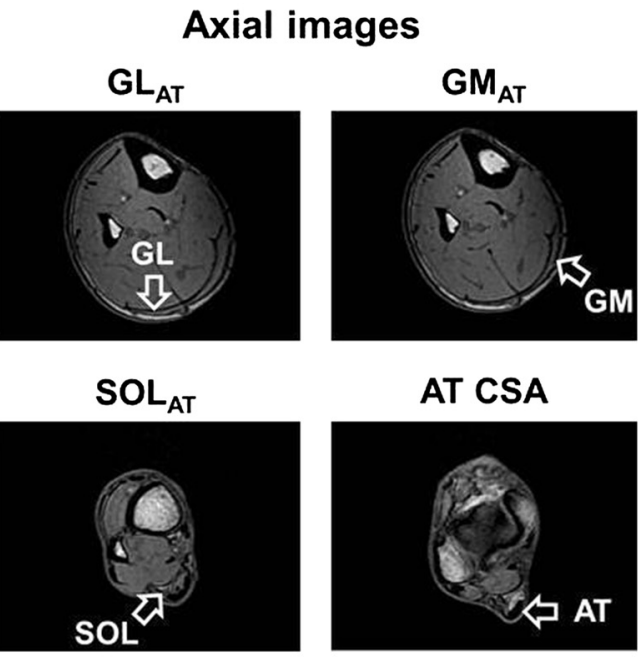

Figure 1 - Representative sagittal and axial magnetic resonance image scans for calculating 3 lengths and CSA of the AT. Both sagittal and axial images were used to measure 3 lengths of the AT. The 3 AT lengths were calculated as the distances from the calcaneal tuberosity to the muscle-tendon junction of the SOL, GM, and GL. An axial image for the AT CSA was at $30 \mathrm{~cm}$ above the distal insertion of the AT. Axial images were used to measure the AT CSA. The AT CSA was calculated as the average of 10, 20, and $30 \mathrm{~mm}$ above the distal insertion of the AT. AT indicates Achilles tendon; CSA, cross-sectional area; GL, gastrocnemius lateralis; GM, gastrocnemius medialis; SOL, soleus. 
Table 1 Physical Characteristics and AT Morphological Variables in Sprinters

\begin{tabular}{lcc}
\hline & Mean (SD) & Range \\
\hline Body height, cm & $175.6(4.9)$ & $165.4-184.3$ \\
Body weight, kg & $66.6(5.1)$ & $56.5-77.8$ \\
Body mass index, $\mathrm{kg} / \mathrm{m}^{2}$ & $21.6(1.2)$ & $19.4-25.1$ \\
SL, cm & $35.9(1.4)$ & $33.4-38.6$ \\
AT length & & \\
SOL $_{\mathrm{AT}}$ length, cm & $7.5(1.2)$ & $5.4-10.5$ \\
$\mathrm{GM}_{\mathrm{AT}}$ length, cm & $20.5(2.0)$ & $16.3-25.8$ \\
$\mathrm{GL}_{\mathrm{AT}}$ length, cm & $21.3(1.9)$ & $16.7-26.0$ \\
Relative AT length & \\
Relative SOL $_{\mathrm{AT}}$ length, \% of SL & $20.9(3.0)$ & $14.7-27.9$ \\
Relative GM $_{\mathrm{AT}}$ length, \% of SL & $57.2(5.6)$ & $46.0-71.5$ \\
Relative $_{\mathrm{AT}}$ length, \% of SL & $59.4(5.4)$ & $46.2-73.4$ \\
AT CSA, mm & & $75.0-154.2$ \\
Relative AT CSA, mm $^{2} / \mathrm{kg}^{2 / 3}$ & $106.4(19.6)$ & $4.5-9.4$ \\
\hline Abbriat & $6.5(1.3)$ &
\end{tabular}

Abbreviations: AT, Achilles tendon; CSA, cross-sectional area; GL, gastrocnemius lateralis; GM, gastrocnemius medialis, SL, shank length; SOL, soleus. Note: Values are presented as mean (SD). Relative AT lengths of the triceps surae were normalized to the SL. The AT CSA was calculated as an average of 10, 20, and $30 \mathrm{~mm}$ above the distal insertion of the AT. Relative AT CSA was normalized to body weight to the two-third power.

\section{Table 2 Correlation Coefficients Between AT Morphological Variables and Personal Best 100-m Sprint Time in Sprinters}

\begin{tabular}{lcc}
\hline & $\boldsymbol{r}$ (lower limit to upper limit) & $\boldsymbol{P}$ value \\
\hline AT length & $.064(-.212$ to .331$)$ & .678 \\
SOL $_{\mathrm{AT}}$ length & $-.023(-.294$ to .252$)$ & .883 \\
$\mathrm{GM}_{\mathrm{AT}}$ length & $.048(-.228$ to .317$)$ & .749 \\
GL $_{\mathrm{AT}}$ length & & \\
Relative AT length & $.102(-.176$ to .365$)$ & .500 \\
Relative SOL & $.096(-.182$ to .359$)$ & .878 \\
Relative $\mathrm{GM}_{\mathrm{AT}}$ length & $.023(-.252$ to .294$)$ & .515 \\
Relative GL $\mathrm{AT}$ length & $.012(-.262$ to .284$)$ & .936 \\
AT CSA & $.084(-.193$ to .349$)$ & .584 \\
Relative AT CSA &
\end{tabular}

Abbreviations: AT, Achilles tendon; CSA, cross-sectional area; GL, gastrocnemius lateralis; GM, gastrocnemius medialis; SOL, soleus.

correlations between 3 AT lengths and AT CSA $(r=.038$ for $\mathrm{SOL}_{\mathrm{AT}}, r=.122$ for $\mathrm{GM}_{\mathrm{AT}}, r=.157$ for $\mathrm{GL}_{\mathrm{AT}}$; all $\left.P \mathrm{~s}>.05\right)$.

Correlation coefficients between AT morphological variables and personal best $100-\mathrm{m}$ sprint time in sprinters are summarized in Table 2 . The absolute 3 AT lengths did not correlate significantly with personal best $100-\mathrm{m}$ sprint time $\left(r=.064\right.$ for $\mathrm{SOL}_{\mathrm{AT}}, r=-.023$ for $\mathrm{GM}_{\mathrm{AT}}, r=.048$ for $\mathrm{GL}_{\mathrm{AT}}$; all $\left.P \mathrm{~s}>.05\right)$. Similarly, no significant correlations were observed between the relative 3 AT lengths and personal best $100-\mathrm{m}$ sprint time $\left(r=.102\right.$ for $\mathrm{SOL}_{\mathrm{AT}}, r=.096$ for $\mathrm{GM}_{\mathrm{AT}}, r=.023$ for $\mathrm{GL}_{\mathrm{AT}}$; all $\left.P \mathrm{~s}>.05\right)$. Additionally, there were no significant correlations between the absolute and relative AT CSAs and personal best $100-\mathrm{m}$ sprint time $(r=.012$ and .084 , respectively, both $P$ s $>.05)$.

\section{Discussion}

One study by Monte and Zamparo ${ }^{8}$ reported that the absolute length of the $\mathrm{GM}_{\mathrm{AT}}$ correlated with sprint performances, including personal best $100-\mathrm{m}$ sprint time. By contrast, the primary finding of the present study was that the absolute and relative AT lengths at 3 different portions of the triceps surae muscle did not correlate with personal best $100-\mathrm{m}$ time in sprinters. Our finding could not corroborate the result of the study by Monte and Zamparo. ${ }^{8} \mathrm{~A}$ possible reason for this discrepancy is that we recruited sprinters who had a mean personal best 100 -m sprint time of $11.12(0.43)$ seconds (range, 10.22-11.86 s), whereas Monte and Zamparo ${ }^{8}$ recruited sprinters who had a mean personal best $100-\mathrm{m}$ sprint time of 10.66 (0.51) seconds (range, 10.02-11.44 s). Thus, our participants were slower than that of the sprinters who participated in Monte and Zamparo's study. ${ }^{8}$ This may explain the discrepancy between the findings of the present and previous studies. Nevertheless, in an additional analysis conducted in the present study, we could not find positive correlations between longer absolute and relative $\mathrm{GM}_{\mathrm{AT}}$ and $\mathrm{GL}_{\mathrm{AT}}$ and better personal best $100-\mathrm{m}$ sprint time in high-level sprinters $(\mathrm{n}=16 ; r=-.099$ to -.281 ; all $P \mathrm{~s}>.05)$, as defined by having a personal best $100-\mathrm{m}$ sprint time faster than 11 seconds (mean, 10.60 [0.20] s) based on previous studies. ${ }^{18,19}$ Furthermore, a significant correlation between shorter relative $\mathrm{SOL}_{\mathrm{AT}}$ and better personal best $100-\mathrm{m}$ sprint time was observed $(r=.574, P=.020)$. A trend against such a significance was also observed between absolute $\mathrm{SOL}_{\mathrm{AT}}$ and personal best 100$\mathrm{m}$ sprint time $(r=.490, P=.054)$. These findings indicate that longer AT may not be associated with better $100-\mathrm{m}$ sprint time in sprinters. Therefore, the discrepancy between the findings of the present study and those of Monte and Zamparo's study ${ }^{8}$ could not be clearly explained by the difference in personal best $100-\mathrm{m}$ sprint time between sprinters recruited in these studies. Taken together, after measuring 3 AT lengths of the triceps surae, we suggest that the AT length may not be related to achieving superior $100-\mathrm{m}$ sprint time in sprinters.

This study measured the AT length using MRI, whereas Monte and Zamparo ${ }^{8}$ measured it using US. MRI is known to be more appropriate for measuring morphological variables than US. ${ }^{9,10}$ The US-measured AT length had high reliability, as determined by Monte and Zamparo ${ }^{8}$ and other previous studies. ${ }^{20-22}$ Nevertheless, the reliability of the AT length measurement appears to be higher for MRI than US. Indeed, CVs of 2 measurements for all 3 AT lengths in the present study were lower than $1 \%$ (eg, $0.3 \%$ for $\mathrm{GM}_{\mathrm{AT}}$ ), whereas the $\mathrm{CV}$ of 2 measurements for the $\mathrm{GM}_{\mathrm{AT}}$ in Monte and Zamparo's study ${ }^{8}$ was $4 \%$. Additionally, in the present study, we examined the reproducibility of 3 AT length measurements on 2 separate days and determined excellent ICCs (-.994) for these AT lengths. ${ }^{23}$ The day-to-day ICCs of the present study were higher than that of previous studies using US. ${ }^{20-22}$ However, Monte and Zamparo ${ }^{8}$ did not examine the day-to-day reliability for the $\mathrm{GM}_{\mathrm{AT}}$. Furthermore, prior to the present study, we calculated the required sample size using a priori analysis to determine the relationship between the morphological variable and the personal best $100-\mathrm{m}$ sprint time in sprinters and found it to be $\geq 29$ participants; thus, the number of participants recruited in the present study $(n=48)$ was sufficient for ensuring statistical power and sensitivity. By contrast, Monte and Zamparo ${ }^{8}$ did not calculate the effective sample size, and the number of participants recruited in their study $(n=18)$ was lower than that calculated in our study. Therefore, these factors may at least partially explain the discrepancy between the findings of the present and previous studies. 
Another primary finding of this study was that the AT CSA did not correlate with personal best $100-\mathrm{m}$ sprint time in sprinters. The AT CSA is known to be larger in endurance runners than in untrained participants ${ }^{16,24,25}$; thus, the larger AT is a specific morphology of endurance runners. Nevertheless, our previous study determined that the AT CSA did not correlate with running performance in endurance runners. ${ }^{7}$ Additionally, in another study, we determined that although the AT CSA was larger in endurance runners than in untrained participants, it did not differ between sprinters and untrained participants ${ }^{16}$; thus, the AT size may not be an important morphological factor of sprinters. However, Monte and Zamparo ${ }^{8}$ reported that the US-measured AT CSA correlated with personal best $100-\mathrm{m}$ sprint time in sprinters. The results of the CSA in addition to length of the AT measured using MRI in the present study could not corroborate the US-measured results obtained by Monte and Zamparo's study. ${ }^{8}$ Previous studies reported the lack of correlation between US- and MRI-measured AT CSAs. ${ }^{26,27}$ Therefore, the discrepancy between the findings of the present study and those of Monte and Zamparo's study ${ }^{8}$ may be attributed to the difference in apparatuses (ie, MRI vs US) used in these studies. Taken together, we suggest that the AT morphological variables, such as the length and CSA, may not be important factors in achieving superior 100 -m sprint time in sprinters.

Previous studies reported that mechanical properties (eg, higher stiffness) of the AT correlated with isometric plantar flexor torque. ${ }^{18,28}$ The increases in the length and CSA of the AT appears to affect its mechanical properties because of increased AT volume ${ }^{29}$; thus, longer and larger AT may be useful in enhancing plantar flexor torque, which may contribute to greater peak vertical ground reaction force and better sprint performance in sprinters. Nevertheless, Kubo et al. ${ }^{18}$ reported that although the AT stiffness correlated with isometric plantar flexor torque in untrained participants, this relationship was absent in sprinters. Furthermore, they determined no correlation between AT stiffness and personal best 100-m sprint time in sprinters. ${ }^{18}$ Therefore, morphological and mechanical variables of the AT may not be required for superior 100-m sprint time in sprinters. Previous studies determined that the plantar flexor torque was increased by changing velocity from walking to running, whereas it remained unchanged when altering velocity from running to sprinting. ${ }^{11,12}$ Furthermore, although the relative contribution of the plantar flexor torque during sprinting may be higher than that of the knee joint torque, this contribution may be lower than that of the hip joint torque. ${ }^{1,11,12,30}$ Therefore, an increase in the plantar flexor torque depending on the AT morphological and mechanical variables may have a little effect on achieving superior 100 -m sprint performance in sprinters. In summary, the findings of the present study may indicate the low contributions of the AT variables and plantar flexor torque during superior 100-m sprinting.

The present study had several limitations. First, we recruited only Japanese male sprinters, and, therefore, it remains unclear whether our findings can be generalized to other racial and ethnic groups or to female sprinters. In particular, the discrepancy between the findings of the present study and those of Monte and Zamparo's study ${ }^{8}$ could be attributed to the different race of the sprinters recruited in these studies (ie, Japanese vs European). Additionally, the length and CSA of the AT in Japanese may be shorter and larger, respectively, than in other races ${ }^{15}$; thus, the genetic factor may have affected to the assessment of the relationships between the AT morphological variables and 100-m sprint performance. Further studies are needed to determine the relationship between the AT morphological variables and 100-m sprint performance in sprinters of different races and sex. Second, although we used only the personal best $100-\mathrm{m}$ sprint time, as a parameter of sprint performance, it inevitably includes various sprinting phases. ${ }^{13,31}$ Monte and Zamparo ${ }^{8}$ reported that the length and CSA of the AT correlated with sprint velocity during a $20-\mathrm{m}$ sprint. Considering their findings, these AT morphological variables may play important roles in achieving superior performance during block start and/or acceleration phases. Previous studies reported that a better block clearance performance was related to higher sprint velocity during the initial phase in a $100-\mathrm{m}$ sprinting. ${ }^{31-33}$ The plantar flexor torque may contribute to the block clearance performance more than other lower limb joint torques. ${ }^{34,35}$ Additionally, the plantar flexor torque may be the greatest source of positive work during the stance phase of maximum acceleration sprinting among all lower limb joint torques. ${ }^{36}$ Further studies are needed to examine the relationships between the AT morphological variables and biomechanical variables (ie, plantar flexor torque) during some phases, especially the block start and acceleration phases, while a 100-m sprinting. Third, we examined only the relationship between the AT morphological variables and 100-m sprint time, and, therefore, it remains unknown whether our findings can be applied to 200- and 400-m sprint times. In particular, our previous studies determined the same favorable morphological variables contributing to 100 - and $400-\mathrm{m}$ sprint performance. ${ }^{13,14,37,38}$ In a recent study, we determined that a higher ratio of the tibia length relative to the femoral length may be a specific favorable morphology of superior sprint performance in $400 \mathrm{~m}$, but not in 100-m specialized sprinters ${ }^{39}$ potentially by achieving long sprinting economically. ${ }^{40}$ Because the AT plays an important role in using elastic energy during the stance phase of human locomotion, the longer and/or larger AT may contribute to energy saving during 400-m sprinting. Further studies are needed to determine the relationships of the AT morphological variables with sprint performance longer than $100 \mathrm{~m}$ in sprinters.

In conclusion, we determined that with the 3 AT length measurements of the triceps surae muscle, the AT length did not correlate with personal best 100-m sprint time. Furthermore, we found no correlation between the AT CSA and the personal best 100-m sprint time. These findings suggest that the AT morphological variables, such as the length and CSA, may not be related to superior $100-\mathrm{m}$ sprint time in male sprinters. We previously reported a positive correlation between longer AT (ie, $\mathrm{GM}_{\mathrm{AT}}$ ) and better running performance in endurance runners. ${ }^{7}$ Therefore, we could find a difference in morphological factors contributing to superior performance between sprinters and endurance runners. In the clinical setting, our studies may help understand an optimal body structure for successful sprinters and endurance runners.

\section{Acknowledgments}

We are grateful to all subjects who gave of their time and effort to participate in this study. This study was supported by Grant-in-Aid for Scientific Research from the Japanese Ministry of Education, Culture, Sports, Science and Technology (no. $15 \mathrm{~K} 16497$ to T.S. and no. $15 \mathrm{H} 03077$ to T.I.).

\section{References}

1. Novacheck TF. The biomechanics of running. Gait Posture. 1998; 7(1):77-95. PubMed ID: 10200378 doi:10.1016/s0966-6362(97) 00038-6

2. Nagahara R, Kanehisa H, Matsuo A, Fukunaga T. Are peak ground reaction forces related to better sprint acceleration performance? Sports Biomech. 2019;24:1-10. PubMed ID: 30676878 doi:10.1080/ 14763141.2018.1560494 
3. Nagahara R, Mizutani M, Matsuo A, Kanehisa H, Fukunaga T. Association of sprint performance with ground reaction forces during acceleration and maximal speed phases in a single sprint. $J$ Appl Biomech. 2018;34(2):104-110. PubMed ID: 28952906 doi:10.1123/ jab.2016-0356

4. Bramble DM, Lieberman DE. Endurance running and the evolution of Homo. Nature. 2004;432(7015):345-352. PubMed ID: 15549097 doi:10.1038/nature03052

5. Mero A, Komi PV, Gregor RJ. Biomechanics of sprint running. A review. Sports Med. 1992;13(6):376-392. PubMed ID: 1615256 doi:10.2165/00007256-199213060-00002

6. Hunter GR, Katsoulis K, McCarthy JP, et al. Tendon length and joint flexibility are related to running economy. Med Sci Sports Exerc. 2011;43(8)1492-1499. PubMed ID: 21266930 doi:10.1249/MSS. 0b013e318210464a

7. Ueno H, Suga T, Takao K, et al. Relationship between Achilles tendon length and running performance in well-trained male endurance runners. Scand J Med Sci Sports. 2018;28(2):446-451. PubMed ID: 28658509 doi:10.1111/sms.12940

8. Monte A, Zamparo P. Correlations between muscle-tendon parameters and acceleration ability in $20 \mathrm{~m}$ sprints. PLoS One. 2019; 14(3):e0213347. PubMed ID: 30849114 doi:10.1371/journal.pone. 0213347

9. Miyatani M, Kanehisa H, Ito M, Kawakami Y, Fukunaga T. The accuracy of volume estimates using ultrasound muscle thickness measurements in different muscle groups. Eur J Appl Physiol. 2004; 91(2-3):264-272. PubMed ID: 14569399 doi:10.1007/s00421-0030974-4

10. Wachi M, Suga T, Higuchi T, et al. Applicability of ultrasonography for evaluating trunk muscle size: a pilot study. J Phys Ther Sci. 2017;29(2):245-249. PubMed ID: 28265150 doi:10.1589/jpts. 29.245

11. Dorn TW, Schache AG, Pandy MG. Muscular strategy shift in human running: dependence of running speed on hip and ankle muscle performance. J Exp Biol. 2012;215(11):1944-1956. PubMed ID: 22573774 doi:10.1242/jeb.064527

12. Schache AG, Blanch PD, Dorn TW, Brown NA, Rosemond D, Pandy MG. Effect of running speed on lower limb joint kinetics. Med Sci Sports Exerc. 2011;43(7):1260-1271. PubMed ID: 21131859 doi:10. 1249/MSS.0b013e3182084929

13. Miyake Y, Suga T, Otsuka M, et al. The knee extensor moment arm is associated with performance in male sprinters. Eur J Appl Physiol. 2017;117(3):533-539. PubMed ID: 28188370 doi:10.1007/s00421017-3557-5

14. Tanaka T, Suga T, Otsuka M, et al. Relationship between the length of the forefoot bones and performance in male sprinters. Scand J Med Sci Sports. 2017;27(12):1673-1680. PubMed ID: 28207966 doi:10. $1111 / \mathrm{sms} .12857$

15. Kunimasa Y, Sano K, Oda T, et al. Specific muscle-tendon architecture in elite Kenyan distance runners. Scand J Med Sci Sports. 2014;24(4):e269-e274. PubMed ID: 26207267 doi:10.1111/sms. 12161

16. Ueno H, Suga T, Miyake Y, et al. Specific adaptations of patellar and Achilles tendons in male sprinters and endurance runners. Transl Sports Med. 2018;1(3):172-179. doi:10.1002/tsm2.21

17. Wiesinger HP, Rieder F, Kösters A, Müller E, Seynnes OR. Are sport-specific profiles of tendon stiffness and cross-sectional area determined by structural or functional integrity? PLoS One. 2016: 11(6):e0158441. PubMed ID: 27362657 doi:10.1371/journal.pone. 0158441

18. Kubo K, Ikebukuro T, Yata H, Tomita M, Okada M. Morphological and mechanical properties of muscle and tendon in highly trained sprinters. J Appl Biomech. 2011;27(4):336-344. PubMed ID: 21896950 doi:10.1123/jab.27.4.336

19. Kumagai K, Abe T, Brechue WF, Ryushi T, Takano S, Mizuno M. Sprint performance is related to muscle fascicle length in male $100-\mathrm{m}$ sprinters. J Appl Physiol. 2000;88:811-816. PubMed ID: 10710372 doi:10.1152/jappl.2000.88.3.811

20. Brouwer EF, Myhrvold SB, Benth JŠ, Hoelsbrekken SE. Ultrasound measurements of Achilles tendon length using skin markings are more reliable than extended-field-of-view imaging. Knee Surg Sports Traumatol Arthrosc. 2018;26(7):2088-2094. PubMed ID: 29185003 doi:10.1007/s00167-017-4815-0

21. Fouré A, Nordez A, Cornu C. Plyometric training effects on Achilles tendon stiffness and dissipative properties. J Appl Physiol. 2010; 109(3):849-854. PubMed ID: 20576842 doi:10.1152/japplphysiol. 01150.2009

22. Silbernagel KG, Shelley K, Powell S, Varrecchia S. Extended field of view ultrasound imaging to evaluate Achilles tendon length and thickness: a reliability and validity study. Muscles Ligaments Tendons J. 2016;6(1):104-110. PubMed ID: 27331037 doi:10.11138/ mltj/2016.6.1.104

23. Cicchetti DV. Guidelines, criteria, and rules of thumb for evaluating normed and standardized assessment instruments in psychology. Psychol Assess. 1994;6:284-290. doi:10.1037/1040-3590.6.4.284

24. Magnusson SP, Kjaer M. Region-specific differences in Achilles tendon cross-sectional area in runners and non-runners. Eur J Appl Physiol. 2003;90(5-6):549-553. PubMed ID: 12905044 doi:10. 1007/s00421-003-0865-8

25. Rosager S, Aagaard P, Dyhre-Poulsen P, Neergaard K, Kjaer M, Magnusson SP. Load-displacement properties of the human triceps surae aponeurosis and tendon in runners and non-runners. Scand $J$ Med Sci Sports. 2002;12(2):90-98. PubMed ID: 12121426 doi:10. 1034/j.1600-0838.2002.120205.x

26. Bohm S, Mersmann F, Schroll A, Mäkitalo N, Arampatzis A. Insufficient accuracy of the ultrasound-based determination of Achilles tendon cross-sectional area. J Biomech. 2016;49(13):2932-2937. PubMed ID: 27498950 doi:10.1016/j.jbiomech.2016.07.002

27. Kruse A, Stafilidis S, Tilp M. Ultrasound and magnetic resonance imaging are not interchangeable to assess the Achilles tendon crosssectional-area. Eur J Appl Physiol. 2017;117(1):73-82. PubMed ID: 27838848 doi:10.1007/s00421-016-3500-1

28. Muraoka T, Muramatsu T, Fukunaga T, Kanehisa H. Elastic properties of human Achilles tendon are correlated to muscle strength. J Appl Physiol. 2005;99(2):665-669. PubMed ID: 15790689 doi:10. 1152/japplphysiol.00624.2004

29. Bohm S, Mersmann F, Arampatzis A. Human tendon adaptation in response to mechanical loading: a systematic review and metaanalysis of exercise intervention studies on healthy adults. Sports Med Open. 2015;1(1):7. PubMed ID: 27747846 doi:10.1186/s40798015-0009-9

30. Kulmala JP, Korhonen MT, Ruggiero L, et al. Walking and running require greater effort from the ankle than the knee extensor muscles. Med Sci Sports Exerc. 2016;48(11):2181-2189. PubMed ID: 27327033 doi:10.1249/MSS.0000000000001020

31. Otsuka M, Kurihara T, Isaka T. Effect of a wide stance on block start performance in Sprint running. PLoS One. 2015;10(11):e0142230. PubMed ID: 26544719 doi:10.1371/journal.pone.0142230

32. Macadam P, Nuell S, Cronin JB, et al. Kinematic and kinetic differences in block and split-stance standing starts during $30 \mathrm{~m}$ sprint-running. Eur J Sport Sci. 2019;19(8):1024-1031. PubMed ID: 30732539 doi:10.1080/17461391.2019.1575475

33. Nagahara R, Gleadhill S, Ohshima Y. Improvement in sprint start performance by modulating an initial loading location on the starting 
blocks [published online ahead of print July 1, 2020]. J Sports Sci. PubeMed ID: 32608346 doi:10.1080/02640414.2020.1787698

34. Brazil A, Exell T, Wilson C, Willwacher S, Bezodis I, Irwin G. Lower limb joint kinetics in the starting blocks and first stance in athletic sprinting. J Sports Sci. 2017;35(16):1629-1635. PubMed ID: 27598715 doi:10.1080/02640414.2016.1227465

35. Sado N, Yoshioka S, Fukashiro S. Three-dimensional kinetic function of the lumbo-pelvic-hip complex during block start. PLoS One. 2020; 15(3):e0230145. PubMed ID: 32163481 doi:10.1371/journal.pone. 0230145

36. Schache AG, Lai AKM, Brown NAT, Crossley KM, Pandy MG. Lower-limb joint mechanics during maximum acceleration sprinting. J Exp Biol. 2019;222(22):jeb209460. PubMed ID: 31672729 doi:10. 1242/jeb. 209460

37. Tomita D, Suga T, Tanaka T, et al. A pilot study on the importance of forefoot bone length in male 400-m sprinters: is there a key morphological factor for superior long sprint performance? BMC Res Notes. 2018;11(1):583. PubMed ID: 30103812 doi:10.1186/s13104018-3685-y

38. Tomita D, Suga T, Ueno H, et al. Relationship between knee extensor moment arm and long-sprint performance in male 400m sprinters. Transl Sports Med. 2018;1(4):172-179. doi:10.1002/ tsm 2.33

39. Tomita D, Suga T, Terada M, et al. A pilot study on a potential relationship between leg bone length and sprint performance in sprinters; are there any event-related differences in 100-m and 400-m sprints? BMC Res Notes. 2020;13(1):297. PubMed ID: 32571392 doi:10.1186/ s13104-020-05140-Z

40. Hanon C, Gajer B. Velocity and stride parameters of world-class 400meter athletes compared with less experienced runners. J Strength Cond Res. 2009;23(2):524-531. PubMed ID: 19209080 doi:10.1519/ JSC.0b013e318194e071 